\title{
Accelerated Disease Progression after Discontinuation of Sorafenib in a Patient with Metastatic Papillary Thyroid Cancer
}

\author{
Kyung-Jin Yun, Woohyeon Kim, Eun Hee Kim, Min-Hee Kim, Dong-Jun Lim, Moo-Il Kang, Bong-Yun Cha \\ Division of Endocrinology and Metabolism, Department of Internal Medicine, The Catholic University of Korea College of \\ Medicine, Seoul, Korea
}

Distant metastases from papillary thyroid carcinoma (PTC) are rare and are associated with a poor prognosis. Here, we describe a patient with metastatic PTC who was treated with a tyrosine kinase inhibitor (TKI, sorafenib) for several months that was acutely exacerbated by discontinuation. A 43-year-old male was diagnosed with PTC in February 2004 and underwent total thyroidectomy followed by two courses of high-dose radioactive iodine (RAI) therapy. Despite two additional courses of high-dose RAI therapy, lung and muscle metastases were developed. Treatment with sorafenib was begun in September 2010. After 11 months treatment of sorafenib, newly developed metastatic lesions were found in mediastinal lymph nodes, liver, and bones. Considered as treatment failure, the administration of sorafenib was discontinued. Two weeks after sorafenib treatment was stopped, the disease progressed abruptly and caused death of the patient by respiratory failure. In our patient, PTC progressed rapidly after the cessation of sorafenib treatment. Patients with several other types of cancer have also experienced such rapid disease progression, termed "flare-ups." Physicians should be aware that flare-ups may occur in advanced PTC patients following the cessation of TKI therapy.

Keywords: Thyroid neoplasms; Papillary; Neoplasm metastasis; Sorafenib

\section{INTRODUCTION}

Distant metastases from papillary thyroid carcinoma (PTC) are rare and are associated with a poor prognosis [1]. In general, the progression of metastatic PTC is unlikely to be rapid despite a low overall survival rate, especially among young patients [2]. The conventional therapy for metastatic PTC include total thyroidectomy, the removal of resectable metastatic lesions, radioactive iodine (RAI) and/or external beam radiation

Received: 27 June 2013, Revised: 14 August 2013,

Accepted: 4 September 2013

Corresponding author: Min-Hee Kim

Division of Endocrinology and Metabolism, Department of Internal Medicine,

Seoul St. Mary's Hospital, The Catholic University of Korea College of

Medicine, 222 Banpo-daero, Seocho-gu, Seoul 137-701, Korea

Tel: +82-2-2258-6068, Fax: + 82-2-599-3589, E-mail: benedict@catholic.ac.kr at the sites of metastases [3]. If the disease does not respond to these conventional therapies, the use of targeted therapeutic agents such as tyrosine kinase inhibitors (TKIs) is recommended based on the results of clinical trials [3].

Sorafenib (Nexavar, Onyx Pharmaceuticals, Emeryville, CA, USA; and Bayer Healthcare, Wayne, NJ, USA) is a multiple TKI used in the treatment of renal cell carcinoma and hepatocellular carcinoma. By inhibiting both cell surface tyrosine kinase receptors (e.g., vascular endothelial growth factor

\section{Copyright () 2014 Korean Endocrine Society}

This is an Open Access article distributed under the terms of the Creative Commons Attribution Non-Commercial License (http://creativecommons.org/ licenses/by-nc/3.0/) which permits unrestricted non-commercial use, distribution, and reproduction in any medium, provided the original work is properly cited. 
[VEGF] receptors) and intracellular serine/threonine kinases (e.g., BRAF), sorafenib produces antiproliferative and antiangiogenic effects [4]. Because differentiated thyroid cancers have been well documented to exhibit genetic alterations in the mitogen-activated protein kinase (MAPK) pathway, including BRAF mutations [5] and VEGF overexpression [6], sorafenib has been proposed as a good candidate therapy for refractory thyroid cancers. Recently, several reports have demonstrated substantial efficacy of sorafenib in the treatment of refractory thyroid cancers $[2,7]$.

Here, we report sorafenib therapy for a patient with RAI-refractory metastatic PTC. Despite the use of sorafenib and the maintenance of a partial response for several months, the disease progressed, producing new metastatic lesions in the lymph nodes, liver, and bone. After the cessation of sorafenib treatment due to disease progression, the growth of the disease, including lesions stabilized under sorafenib use, was further accelerated. The objective of this report is to highlight the rapid progression of PTC after the cessation of sorafenib treatment.

\section{CASE REPORT}

A 43-year-old male patient presented with metastatic PTC. He had been diagnosed with PTC in February 2004 and underwent total thyroidectomy at another hospital. High-dose $(150 \mathrm{mCi})$ RAI therapy was performed twice in May 2004 and July 2006. In April 2007, right neck lymph node dissection was performed due to the discovery of recurrent PTC in cervical lymph nodes. Despite the application of additional high-dose RAI therapy in August 2007, hematogenous lung metastasis was observed in April 2009, and a fourth course of RAI therapy was performed in July 2009.

Nevertheless, chest computed tomography (CT) in February 2010 showed interval increase in the size of multiple hematogeneous metastasis in both lungs. For the further treatment, he was transferred to our hospital. On the basis of chest CT scans (Fig. 1A) performed in August 2010, the disease was classified as progressive disease according to the Response Evaluation Criteria in Solid Tumors (RECIST) criteria. Additionally, two measurable lesions in the right gluteus muscle (Fig. 1B) were confirmed to be metastatic PTCs by ultrasound-guided needle biopsies (Fig. 2). Increase in serum thyroglobulin (Tg) level was also noted after he was transferred to our institution. Considering changes in both imaging studies and serum $\mathrm{Tg}$ level after four cycles of RAI therapy, the disease was considered as refractory to RAI therapy. Therefore, the patient was recruited for an investigational study of sorafenib, which had been approved by the institutional review board, and received his first medication in September 2010.

After 2 months of sorafenib administration $(800 \mathrm{mg}$ per day), a follow-up chest CT scan showed slight decreases in metastatic lung and muscle lesions compared with previous examinations (Fig. 1C, D). Because there was no evidence of disease progression or newly developed lesions revealed by imaging studies, which were repeated every 2 months, sorafenib was maintained at a full dose.

Until August 2011, after 11 months of sorafenib administration, multiple metastatic lesions in lung parenchyma and in the right gluteus muscle showed little interval change. However, chest CT scan revealed marked increase in the size of the subcarinal and right interlobar nodes, which had previously been stable, and a newly enlarged right paratracheal lymph node. In follow-up chest and abdominal CT in October 2011, though multiple metastatic nodules in both lungs showed stabilization in size, progression of mediastinal lymph nodes and multiple new hepatic metastatic masses were identified. In addition, a lumbar spine magnetic resonance imaging scan, obtained due to back pain, revealed extensive metastatic lesions of thoracic spine, lumbar spine, sacrum, and pelvis, which were not identified in previous chest or abdominal CT scans. Because the disease had progressed, palliative treatments, such as external beam radiation of the lumbar spine, were planned.

On October 8, 2011, radiotherapy of the metastatic lesions of the lumbar spine and the sacrum was initiated. In addition, sorafenib was discontinued on October 17, 2011. After the first fraction of radiotherapy, the patient visited an emergency room for dyspnea and fever on November 2011, after 17 days sorafenib discontinuation. A chest CT showed marked progression of metastatic lesions in the lungs, which had been stable under sorafenib, and the development of pleural metastasis with bilateral effusion (Fig. 1E). To relieve the symptoms, the pleural effusions were drained using a pigtail catheter. On the 12th day of hospitalization, a liver biopsy was performed for further evaluation and management. The histopathology, which was supported by immunohistochemical staining (Fig. 3), was consistent with the anaplastic transformation of follicular cell-derived carcinoma [8]. In addition, BRAF V600E mutation was found in the tissue.

CT pulmonary angiography performed on the 13th hospital day revealed marked progression of lung, pleural, and mediastinal metastases compared with a chest CT scan obtained 2 weeks prior. No pulmonary thromboembolism was identified. 

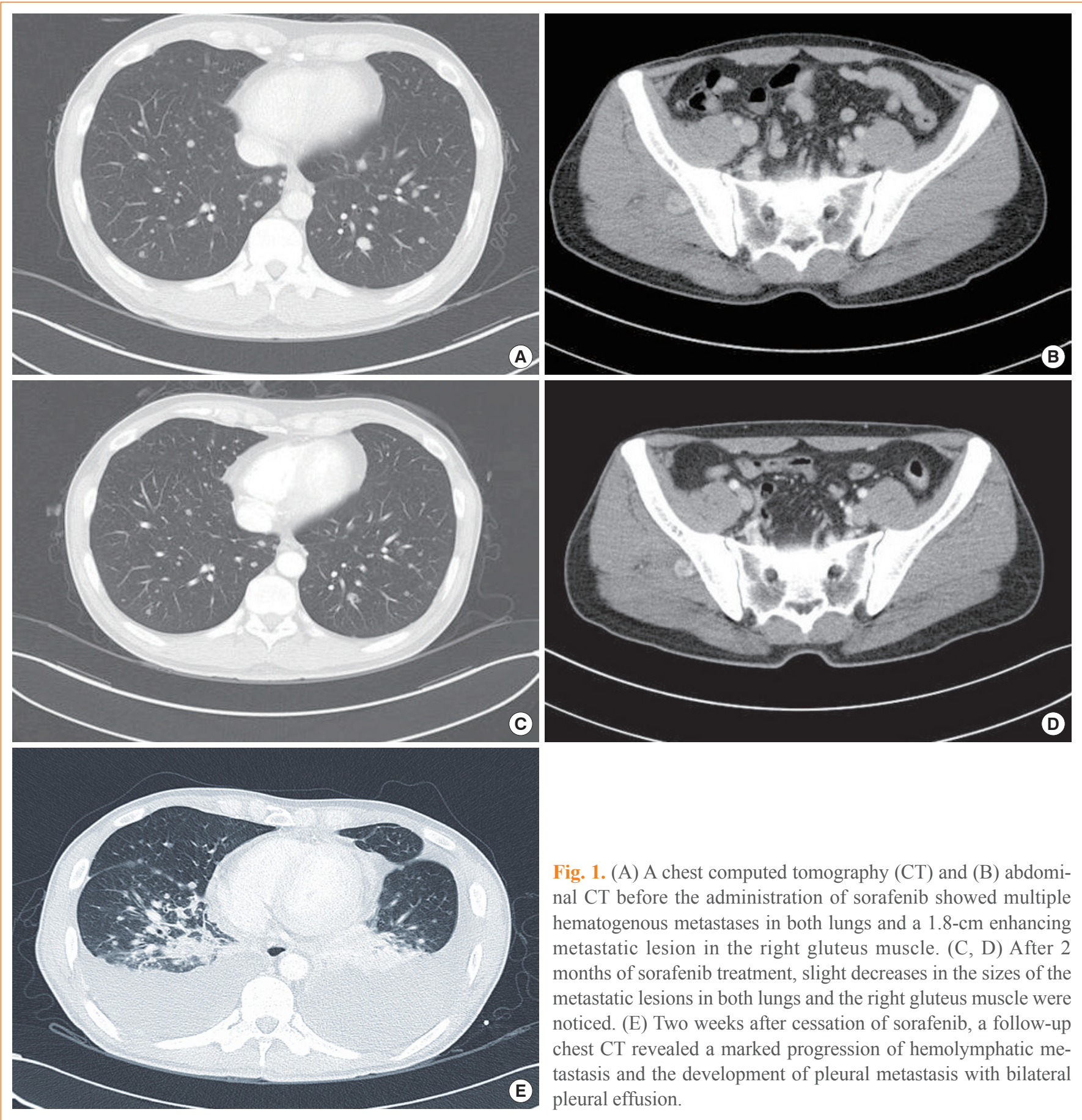

Fig. 1. (A) A chest computed tomography (CT) and (B) abdominal CT before the administration of sorafenib showed multiple hematogenous metastases in both lungs and a 1.8 -cm enhancing metastatic lesion in the right gluteus muscle. (C, D) After 2 months of sorafenib treatment, slight decreases in the sizes of the metastatic lesions in both lungs and the right gluteus muscle were noticed. (E) Two weeks after cessation of sorafenib, a follow-up chest CT revealed a marked progression of hemolymphatic metastasis and the development of pleural metastasis with bilateral pleural effusion.

The patient died on the 14th day of hospitalization due to respiratory failure.

\section{DISCUSSION}

PTC has been reported to initially present with distant metastases in approximately 4\% of cases [9]. According to the 2009
American Thyroid Association guidelines [3], the removal of resectable metastatic disease and repeated RAI therapy are the mainstay of further management in cases of RAI-avid lesions.

There are insufficient data showing the efficacy of RAI therapy for metastatic thyroid cancers. It has been reported that a substantial number of metastatic thyroid carcinomas would not be responsive to RAI therapy because $20 \%$ to $50 \%$ lose the 

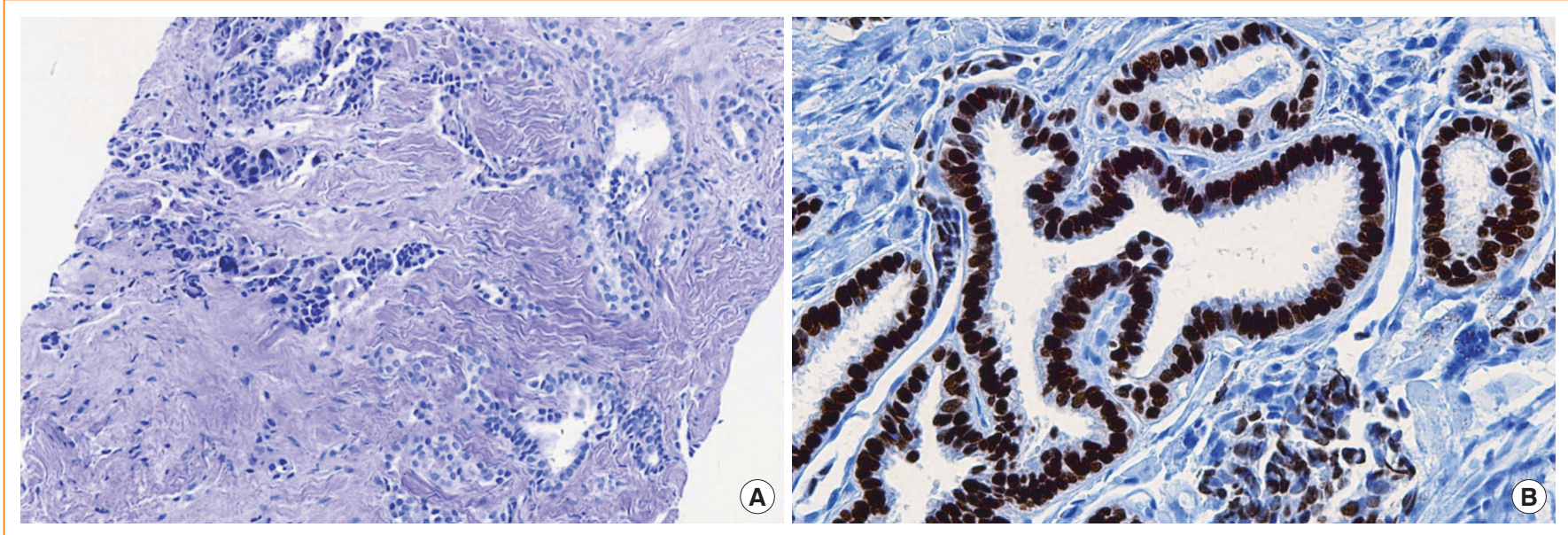

Fig. 2. (A) A histopathological examination of the right gluteus muscle lesion showed papillary architecture (H\&E stain, $\times 200)$. (B) Immunohistochemical staining showed strong thyroid transcription factor-1 immunoreactivity $(\times 400)$.
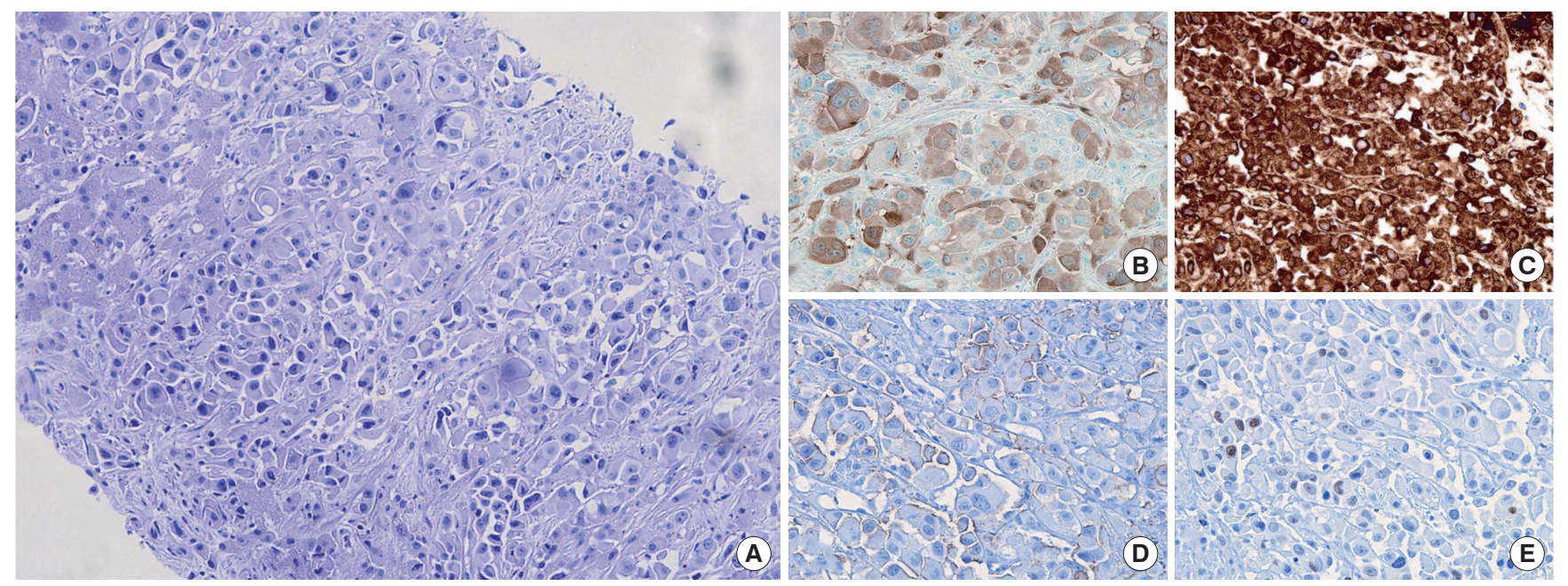

Fig. 3. (A) A histopathological examination of the liver revealed the anaplastic transformation of follicular cell-derived thyroid carcinoma (H\&E stain, $\times 200)$. (B) Immunohistochemical staining showed strong immunoreactivity with CK-7 (×400), (C) galectin-3 (×400), (D) human mesothelial cell-1 $(\times 400)$, and $(\mathrm{E})$ thyroid transcription factor-1 $(\times 400)$.

ability to concentrate iodine [10]. In addition, it is known that thyroid carcinomas lose RAI avidity during dedifferentiation [11]. The current recommendation for patients with PTC who do not respond to conventional therapy is participation in a clinical trial using targeted therapy agents such as TKIs.

Our patient, whose disease was refractory to RAI therapy, was a good candidate for TKI therapy. Treating the patient with sorafenib for 2 months achieved $13.1 \%$ reduction in the total sum size of measurable lesions (stable disease according to the RECIST criteria). Furthermore, after the initial partial response, no significant changes in the lesions were found for 9 months.
However, sorafenib was stopped 13 months later because newly developed liver and bone metastases and the progression of existing lesions in lymph nodes were observed. As soon as sorafenib was discontinued, the disease progressed rapidly. Prior studies of patients with several advanced cancers showed the cessation of TKI therapy was followed by the rapid progression of disease or mortality $[12,13]$. Several reports described this as "flare-ups" [12-14]. No definite mechanism for this phenomenon has yet been elucidated. One possible mechanism is the rapid growth of TKI-sensitive clones following the discontinuation of the drug [12,13]. In our case, as shown in Table 1, stabilized lesions in the lungs and the right gluteus 
Table 1. Size Changes of Metastatic Lesions according to Location

\begin{tabular}{lccc}
\hline & 2 mo after sorafenib use & 13 mo after sorafenib use & 2 wk after sorafenib discontinuation \\
\hline Lung (parenchymal) & Decreased & Stable & Increased \\
Lymph node (mediastinal, interlobar) & Stable & Increased & Increased \\
Right gluteus muscle & Decreased & Stable & Increased \\
Liver & Not detected & Detected & Increased \\
Spine & Not detected & Detected & Increased \\
\hline
\end{tabular}

muscle under TKI treatment, which were considered as TKIsensitive, showed rapid progression after cessation of the drug. Therefore, rapid growth of TKI-sensitive clones might play a role in abrupt exacerbation of disease after discontinuation of the drug. Another possible explanation is that cessation of therapy could result in removal of the residual inhibitory effect of the antiangiogenics, regardless of the TKI sensitivity of the lesion in each tissue (lung, lymph node, muscle, liver, or bone), as suggested by another study [14].

Another interesting finding of the patient was the presence of metastatic lesions in the liver, which indicated the anaplastic transformation of thyroid carcinoma. There have been several reports of the anaplastic transformation of PTC in metastatic lesions $[15,16]$, most of which were discovered after RAI therapy. However, the anaplastic transformation in our patient developed 27 months after the final RAI treatment. Thus, considering the time interval between the final RAI treatment and development of liver metastasis, the role of RAI therapy in transformation is not clear in this case. Because the patient was treated with sorafenib, unlike the previous cases, the role of TKIs in anaplastic changes should be considered. Because sorafenib inhibits the MAPK pathway, it is possible that an alternative pathway, such as the AKT pathway, might be activated instead. Activation of the AKT pathway is known to be associated with anaplastic carcinoma [17].

In this context, a multitargeted therapy directed at the inhibition of multiple signaling pathways, including escape and alternate pathways, should be considered not only for effective treatment, but also for the reduction of drug resistance [18]. A therapy to block dual cell proliferation pathways would be promising, such as the combination of a pan-RAF inhibitor and BEZ235 (a dual phosphatidylinositol-4,5-bisphosphate 3-kinase/mammalian target of rapamycin [mTOR] inhibitor) or the combination of mTOR and MEK inhibitors for treating thyroid cancer $[19,20]$.

In conclusion, doctors should be aware of the possibility of flare-ups after the cessation of sorafenib. Additionally, consid- ering the oncogenic variability and complex positive and negative feedback regulation involved in the signal pathways of cancer cells, the activation of an alternative pathway should be taken into account when targeted therapies are administered to RAI-refractory patients.

\section{CONFLICTS OF INTEREST}

No potential conflict of interest relevant to this article was reported.

\section{REFERENCES}

1. Hoie J, Stenwig AE, Kullmann G, Lindegaard M. Distant metastases in papillary thyroid cancer. A review of 91 patients. Cancer 1988;61:1-6.

2. Gupta-Abramson V, Troxel AB, Nellore A, Puttaswamy K, Redlinger M, Ransone K, Mandel SJ, Flaherty KT, Loevner LA, O'Dwyer PJ, Brose MS. Phase II trial of sorafenib in advanced thyroid cancer. J Clin Oncol 2008;26:4714-9.

3. American Thyroid Association (ATA) Guidelines Taskforce on Thyroid Nodules and Differentiated Thyroid Cancer, Cooper DS, Doherty GM, Haugen BR, Kloos RT, Lee SL, Mandel SJ, Mazzaferri EL, McIver B, Pacini F, Schlumberger M, Sherman SI, Steward DL, Tuttle RM. Revised American Thyroid Association management guidelines for patients with thyroid nodules and differentiated thyroid cancer. Thyroid 2009;19:1167-214.

4. Kapiteijn E, Schneider TC, Morreau H, Gelderblom H, Nortier JW, Smit JW. New treatment modalities in advanced thyroid cancer. Ann Oncol 2012;23:10-8.

5. Xing M, Westra WH, Tufano RP, Cohen Y, Rosenbaum E, Rhoden KJ, Carson KA, Vasko V, Larin A, Tallini G, Tolaney S, Holt EH, Hui P, Umbricht CB, Basaria S, Ewertz M, Tufaro AP, Califano JA, Ringel MD, Zeiger MA, Sidransky D, Ladenson PW. BRAF mutation predicts a poorer clinical prognosis for papillary thyroid cancer. J Clin En- 
docrinol Metab 2005;90:6373-9.

6. Fenton C, Patel A, Dinauer C, Robie DK, Tuttle RM, Francis GL. The expression of vascular endothelial growth factor and the type 1 vascular endothelial growth factor receptor correlate with the size of papillary thyroid carcinoma in children and young adults. Thyroid 2000;10:349-57.

7. Hoftijzer H, Heemstra KA, Morreau H, Stokkel MP, Corssmit EP, Gelderblom H, Weijers K, Pereira AM, Huijberts M, Kapiteijn E, Romijn JA, Smit JW. Beneficial effects of sorafenib on tumor progression, but not on radioiodine uptake, in patients with differentiated thyroid carcinoma. Eur J Endocrinol 2009;161:923-31.

8. Wiseman SM, Griffith OL, Deen S, Rajput A, Masoudi H, Gilks B, Goldstein L, Gown A, Jones SJ. Identification of molecular markers altered during transformation of differentiated into anaplastic thyroid carcinoma. Arch Surg 2007; 142:717-27.

9. Shaha AR, Ferlito A, Rinaldo A. Distant metastases from thyroid and parathyroid cancer. ORL J Otorhinolaryngol Relat Spec 2001;63:243-9.

10. Schlumberger M, Tubiana M, De Vathaire F, Hill C, Gardet P, Travagli JP, Fragu P, Lumbroso J, Caillou B, Parmentier C. Long-term results of treatment of 283 patients with lung and bone metastases from differentiated thyroid carcinoma. J Clin Endocrinol Metab 1986;63:960-7.

11. Robbins RJ, Wan Q, Grewal RK, Reibke R, Gonen M, Strauss HW, Tuttle RM, Drucker W, Larson SM. Real-time prognosis for metastatic thyroid carcinoma based on 2-[18F] fluoro-2-deoxy-D-glucose-positron emission tomography scanning. J Clin Endocrinol Metab 2006;91:498-505.

12. Chaft JE, Oxnard GR, Sima CS, Kris MG, Miller VA, Riely GJ. Disease flare after tyrosine kinase inhibitor discontinuation in patients with EGFR-mutant lung cancer and acquired resistance to erlotinib or gefitinib: implications for clinical trial design. Clin Cancer Res 2011;17:6298-303.

13. Wolter P, Beuselinck B, Pans S, Schoffski P. Flare-up: an often unreported phenomenon nevertheless familiar to oncologists prescribing tyrosine kinase inhibitors. Acta Oncol 2009;48:621-4.

14. Desar IM, Mulder SF, Stillebroer AB, van Spronsen DJ, van der Graaf WT, Mulders PF, van Herpen CM. The reverse side of the victory: flare up of symptoms after discontinuation of sunitinib or sorafenib in renal cell cancer patients. A report of three cases. Acta Oncol 2009;48:927-31.

15. Sotome K, Onishi T, Hirano A, Nakamaru M, Furukawa A, Miyazaki H, Morozumi K, Tanaka Y, Iri H, Mimura Y. A rare case of anaplastic transformation within the metastatic site of the retroperitoneal region in a patient 17 years after total thyroidectomy for papillary carcinoma of the thyroid beginning with multiple bone metastases. Thyroid 2007; 17:1309-11.

16. Al-Qsous W, Miller ID. Anaplastic transformation in lung metastases of differentiated papillary thyroid carcinoma: an autopsy case report and review of the literature. Ann Diagn Pathol 2010;14:41-3.

17. Sherman SI. Targeted therapies for thyroid tumors. Mod Pathol 2011;24 Suppl 2:S44-52.

18. Gossage L, Eisen T. Targeting multiple kinase pathways: a change in paradigm. Clin Cancer Res 2010;16:1973-8.

19. Jin N, Jiang T, Rosen DM, Nelkin BD, Ball DW. Synergistic action of a RAF inhibitor and a dual PI3K/mTOR inhibitor in thyroid cancer. Clin Cancer Res 2011;17:6482-9.

20. Liu D, Xing J, Trink B, Xing M. BRAF mutation-selective inhibition of thyroid cancer cells by the novel MEK inhibitor RDEA119 and genetic-potentiated synergism with the mTOR inhibitor temsirolimus. Int J Cancer 2010;127: 2965-73. 